\title{
THE MEHLER-FOCK TRANSFORM OF GENERAL ORDER AND ARBITRARY INDEX AND ITS INVERSION
}

\author{
CYRIL NASIM \\ Department of Mathematics and Statistics \\ The University of Calgary \\ Calgary, Alberta, Canada \\ (Received July 24, 1983)
}

ABSTRACT. An integral transform involving the associated Legendre function of zero order, $P_{-\frac{1}{2}+i \tau}(x), x \in[1, \infty)$, as the kernel (considered as a function of $\tau$ ), is called Mehler-Fock transform. Some generalizations, involving the function $P_{-\frac{1}{2}+i \tau}^{\mu}(x)$, where the order $\mu$ is an arbitrary complex number, including the case when $\mu=0,1,2, \ldots$, have been known for some time. In this present note, we define a general Mehler-Fock transform involving, as the kernel, the Legendre function $P_{-\frac{1}{2}+t}^{\mu}(x)$, of general order $\mu$ and an arbitrary index $-\frac{1}{2}+t, t=\sigma+i \tau,-\infty<\tau<\infty$. Then we develop a symmetric inversion formulae for these transforms. Many well-known results are derived as special cases of this general form. These transforms are widely used for solving many axisymmetric potential problems.

KEY WORDS AND PHRASES. Mehler-Fock transform, Kontorovich-Lebedev transforms, Legendre functions of first and second kinds, Macdonald function, Bessel functions. 1980 MATHEMATICS SUBJECT CLASSIFICATION CODE. $\quad 44$ A15

\section{INTRODUCTION.}

To solve many axisymmetric potential problems, integral transforms involving associated Legendre functions as kernels, are used widely. When the associated Legendre function of the first kind, $P_{-\frac{1}{2}+i \tau}(x), x \in[1, \infty)$, is used as the kernel, the transform is called Mehler-Fock transform of order zero, [1, p.175(8,9)], and index $-\frac{1}{2}+i \tau,-\infty<\tau<\infty$. Some generalizations of Mehler-Fock transformation involve more general associated Legendre function, namely, $P_{-\frac{1}{2}+i \tau}^{m}(x), m$, a non-negative integer $[2, p .390]$ and $P_{-\frac{1}{2}+i \tau}^{\mu}(x)$, where $\mu \neq 0[3]$, have been known for some time, [cf 4]. In all these generalizations, the emphasis has been to generalize the order $\mu$ of the Legendre function $P_{\nu}^{\mu}(x)$, but no attempt has been made to extend the results for an arbitrary index $v$. In this note we define transforms, involving a more general form of the kernel function, the associated Legendre function of the first kind $P_{-\frac{1}{2}+t}^{\mu}(x)$, with index $t=\sigma+i \tau,-\infty<\tau<\beta$ and $\mu$ an arbitrary complex number. An inversion formula is developed, also, involving the Legendre function $P_{-\frac{1}{2}+t}^{\mu}(x)$ as 
kernel, thus establishing a symmetric inversion theory for general Mehler-Fock transformation. Also, a Parseval type relation for these general transforms is produced. All the well-known results are derived as special cases.

2. THE PRELIMINARY RESULTS.

We note below, for future reference, some of the properties of the associated Legendre function of the first kind, $P_{\nu}^{\mu}(z)$ of order $\mu$ and index $\nu$ for unrestricted $\nu, \mu$ and $z[1, I I I]$. The function is one-valued and regular in the complex plane supposed cut along the real axis from 1 to $-\infty$.

$$
\begin{aligned}
& P_{\nu}^{\mu}(z)=P_{-v-1}^{\mu}(z) \\
& P_{v}^{m}(z)=\frac{\Gamma(v+m+1)}{\Gamma(\nu-m+1)} P_{\nu}^{-m}(z), \quad m=0,1,2, \ldots
\end{aligned}
$$

An important case is when $\mu=0$ and $\nu$ is an non-negative integer. Then

$$
P_{n}(x)=\frac{1}{2^{n} n !} \frac{d^{n}}{d x^{n}}\left(x^{2}-1\right)^{n}
$$

the Legendre polynomial.

Now, consider the representation, [1, p.128 (28)],

$$
\begin{aligned}
& P_{\nu}^{\mu}(z)=2^{\mu}\left(z^{2}-1\right)^{-\frac{1}{2} \mu} \frac{\left(z+\sqrt{z^{2}-1}\right)^{\nu+\mu}}{\Gamma(1-\mu)}{ }_{2} F_{1}\left(-\nu-\mu, \frac{1}{2}-\mu ; 1-2 \mu ; \frac{2 \sqrt{z^{2}-1}}{z+\sqrt{z^{2}-1}}\right) \\
& \operatorname{Re} z>1 \text { and } \operatorname{Re} \mu<1 .
\end{aligned}
$$

Also, [2,p.78(1)],

$$
\begin{array}{r}
{ }_{2} F_{1}(a, b ; c ; z)=\frac{\Gamma^{2}(c)}{\Gamma(a) \Gamma(b) \Gamma(c-a) \Gamma(c-b)} \int_{0}^{1} \tau^{a-1}(1-\tau)^{c-a-1} d \tau \\
\times \int_{0}^{1} \frac{t^{b-1}(1-t)^{c-b-1}}{(1-t \tau z)^{c}} d t,
\end{array}
$$

$\operatorname{Re} c>\operatorname{Re} b>0$.

Using the inequality

$$
\frac{1}{(1-t \tau z)^{c}} \leq \frac{1}{(1-z)^{c}}, \quad c>0,
$$

it is clear that

Thus

$$
{ }_{2} F_{1}(a, b ; c ; z) \leq \frac{1}{(1-z)^{c}} \text {. }
$$

whence,

$$
P_{\nu}^{\mu}(z) \leq \frac{2^{\mu}\left(z+\sqrt{z^{2}-1}\right)^{\nu-\mu+1}}{\Gamma(1-\mu)\left(z^{2}-1\right)^{\frac{7}{2} \mu}\left(z-\sqrt{z^{2}-1}\right)^{1-2 \mu}}, \quad \operatorname{Re} \mu<\frac{1}{2},
$$

$$
P_{\nu}^{\mu}(x) \sim x^{\nu} \text { as } \quad x \rightarrow \infty
$$

and

$$
P_{\nu}^{\mu}(x) \sim\left(x^{2}-1\right)^{-\frac{1}{2} \mu} \quad \text { as } x \rightarrow 1+.
$$

Also, that

$$
{ }_{2} F_{1}(\sigma+i \tau, b ; c ; z)=0(1), \quad \text { as }|\tau| \rightarrow \infty,
$$

whence

$$
P_{\sigma+i \tau}^{\mu}(z)=0(1) \quad \text { as }|\tau| \rightarrow \infty,
$$

provided $\operatorname{Re} \mu<\frac{1}{2}$. 
We shall also need the following result, [5, p.75(76)].

Lemma 1.

$$
\text { Let } \begin{aligned}
f(\nu) & =0\left(e^{-v}\right), & & \text { as } v \rightarrow \infty \\
& =0\left(v^{\frac{1}{2}}\right), & & \text { as } \nu \rightarrow 0 .
\end{aligned}
$$

If

$$
f(x)=\frac{1}{\pi i} \int_{\sigma-i \infty}^{\sigma+i \infty} t K_{t}(x) g(t) d t, \quad \alpha>-\frac{1}{2},
$$

then $g(t)=\int_{0}^{\infty} \frac{f(\nu)}{v} I_{t}(\nu) d v$,

where $K_{t}(\nu)$ and $I_{t}(\nu)$ are the Macdonald function and the Bessel function respectively of order $t$, a complex number. Next let us consider the contour integral

$$
I=\int_{C} z K_{z}(u) g(z) d z,
$$

where $C$ is the closed contour in the $z$-plane as shown. Now $K_{z}(u)$ is an entire function of $z$ and it is clear that $g(z)$ is regular inside and on $C$ from (2.7) above.

Thus, by Cauchy's theorem of residues,

$$
I=0 \text {, }
$$

or

$$
\begin{aligned}
0= & i \int_{-\tau}^{\tau}(\sigma+i y) K_{\sigma+i y}(u) g(\sigma+i y) d y \\
& +\int_{\sigma}^{\sigma}(x+i \tau) K_{x+i \tau}(u) g(x+i \tau) d x \\
& +i \int_{\tau}^{-\tau}(-\delta+i y) K_{-\sigma+i y}(u) g(-\sigma+i y) d y \\
& +\int_{-\sigma}^{\sigma}(x-i \tau) K_{x-i \tau}(u) g(x-i \tau) d x \\
= & I_{1}+I_{2}+I_{3}+I_{4}, \text { say, }
\end{aligned}
$$

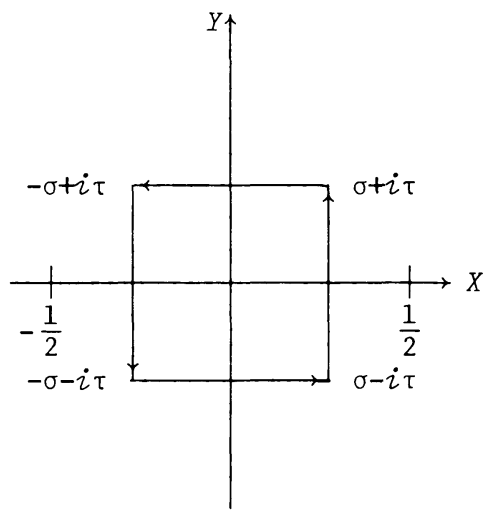

Now the existance of the integral in (2.6), implies that

thus

$$
\left|(x+i \tau) K_{x+i \tau}(u) g(x+i \tau)\right| \rightarrow 0 \quad \text { as }|\tau| \rightarrow \infty \text {, }
$$

$$
\left|I_{2}\right| \text { and }\left|I_{4}\right| \text { both vanish as }|\tau| \rightarrow \infty \text {. }
$$

And we now have,

$$
I_{1}=-I_{3} \text { or }|\tau| \rightarrow \infty
$$

or,

$$
i \int_{-\infty}^{\infty}(\sigma+i y) K_{\sigma+i y}(u) g(\sigma+i y) d y=-i \int_{\infty}^{-\infty}(-\sigma+i y) K_{-\sigma+i y}(u) g(-\sigma+i y) d y
$$

on simplifying, we obtain

$$
\begin{aligned}
\int_{\sigma-i \infty}^{\sigma+i \infty} z K_{z}(u) g(z) d t & =-\int_{\sigma-i \infty}^{\sigma+i \infty} z K_{z}(u) g(-z) d z \\
& =i f(u),
\end{aligned}
$$

using (2.6) and the fact that $K_{-z}(u)=K_{z}(u)$. 
Thus,

$$
f(u)=\frac{1}{2 \pi i} \int_{\sigma-i \infty}^{\sigma+i \infty} z K_{z}(u)[g(z)-g(-z)] d z
$$

From the definition of the function $g$ given by (2.7),

or

$$
g(z)-g(-z)=\int_{0}^{\infty} \frac{f(\nu)}{\nu}\left[I_{z}(\nu)-I_{-z}(\nu)\right] d \nu,
$$

$$
-\frac{\pi}{2} \frac{g(z)-g(-z)}{\sin (\pi z)}=\int_{0}^{\infty} \frac{f(\nu)}{\nu} K_{z}(\nu) d \nu,
$$

If we let

$$
-\frac{\pi}{2} \frac{g(z)-g(-z)}{\sin (\pi z)}=F(z), \text { say, }
$$

then (2.8) and (2.9) reduce to

and

$$
f(u)=\frac{i}{\pi^{2}} \int_{\sigma-i \infty}^{\sigma+i \infty} z \sin (\pi z) K_{z}(u) F(z) d z
$$

$$
F(z)=\int_{0}^{\infty} \frac{f(v)}{v} K_{z}(v) d v,
$$

respectively.

Now we have a generalized Kontorovich-Lebedev transforms in a symmetrical form, (cf. 6]. Thus, we have shown:

Lemma 3. Let $\begin{array}{rlrl}f(v) & =0\left(e^{-v}\right), & \text { as } v \rightarrow \infty \\ & =0\left(v^{\frac{1}{2}}\right), & & \text { as } v \rightarrow 0 .\end{array}$

If

then

$$
f(v)=\frac{i}{\pi^{2}} \int_{\sigma-i \infty}^{\sigma+i \infty} z \sin (\pi z) K_{z}(v) F(z) d z,
$$

$$
F(z)=\int_{0}^{\infty} \frac{f(v)}{v} K_{z}(v) d v
$$

where $|\sigma|<\frac{1}{2}$.

If we set $\sigma=0$, the above pair reduces to

where

$$
f(v)=\frac{2}{\pi^{2}} \int_{0}^{\infty} \tau \sinh (\pi \tau) K_{i \tau}(v) F(i \tau) d \tau
$$

$$
F(i \tau)=\int_{0}^{\infty} \frac{f(v)}{v} K_{i \tau}(v) d v
$$

giving us the usual Kontorovich-Lebedev transformation [2, p.361]

Lemma 4. If $\psi(t) \in L(\sigma-i \infty, \sigma+i \infty),|\sigma|<\frac{1}{2}$, and $\operatorname{Re} \mu<1$,

then

$$
\int_{1}^{\infty} \frac{e^{-x y}}{\left(y^{2}-1\right)^{\mu / 2}} d y \int_{\sigma-i \infty}^{\sigma+i \infty} \psi(t) P_{-\frac{1}{2}+t}^{\mu}(y) d t=\sqrt{\frac{2}{\pi}} x^{\mu-\frac{1}{2}} \int_{\sigma-i \infty}^{\sigma+i \infty} \psi(t) K_{t}(x) d t .
$$

Proof. Consider the double integral

$$
\left|\int_{1}^{\infty} \frac{e^{-x y}}{\left(y^{2}-1\right)^{\mu / 2}} d y \int_{\sigma-i \infty}^{\sigma+i \infty} \psi(t) P_{-\frac{1}{2}+t}^{\mu}(y) d t\right|
$$




$$
\begin{aligned}
& \leq \int_{1}^{\infty} \int_{\sigma-i \infty}^{\sigma+i \infty}\left|\frac{e^{-x y} \psi(t)}{\left(y^{2}-1\right)^{\mu / 2}} P_{-\frac{1}{2}+t}^{\mu}(y)\right| u t d y \\
& =\int_{1}^{\infty} \int_{-\infty}^{\infty} \frac{e^{-x y}}{\left(y^{2}-1\right)^{\mu / 2}} \psi(\sigma+i \tau) P_{-\frac{1}{2}+\sigma+i \tau}^{\mu}(y) \mid d \tau d y \\
& =\int_{1}^{\delta} \int_{-\infty}^{\infty}|\cdot| d \tau d y+\int_{\delta}^{\infty} \int_{-\infty}^{\infty}|\cdot| d \tau d y, \delta>1 . \\
& \leq \int_{1}^{\delta} \int_{-\infty}^{\infty}\left|\frac{e^{-x y}}{\left(y^{2}-1\right)^{\mu / 2}} \psi(\sigma+i \tau)\left(y^{2}-1\right)^{\mu / 2} d \tau d y+\int_{\delta}^{\infty} \int_{-\infty}^{\infty}\right| \frac{e^{-x y}}{\left(y^{2}-1\right)^{\mu / 2}} \psi(\sigma+i \tau) \mid d \tau d y \\
& =\int_{1}^{\delta} \frac{e^{-x y}}{\left(y^{2}-1\right)^{\mu}} d y \int_{-\infty}^{\infty}|\psi(\sigma+i \tau)| d \tau+\int_{\delta}^{\infty} \frac{e^{-x y}}{\left(y^{2}-1\right)^{\mu / 2}} d y \times \\
& \int_{-\infty}^{\infty}|\psi(\sigma+i \tau)| d \tau<\infty \text {, due to the hypotheses. }
\end{aligned}
$$

Hence

$$
\int_{1}^{\infty} \int_{\sigma-i \infty}^{\sigma+i \infty} \frac{e^{-x y}}{\left(y^{2}-1\right)^{\mu / 2}} \psi(t) P_{-\frac{1}{2}+t}^{\mu}(y) d t d y
$$

is absolutely convergent, and therefore the change of order of integration is possible. And,

$$
\begin{aligned}
\int_{1}^{\infty} \frac{e^{-x y}}{\left(y^{2}-1\right)^{\mu / 2}} d y \int_{\sigma-i \infty}^{\sigma+i \infty} \psi(t) P_{-\frac{1}{2}+t}^{\mu}(y) d t \\
=\int_{\sigma-i \infty}^{\sigma+i \infty} \psi(t) d t \int_{1}^{\infty} \frac{e^{-x y}}{\left(y^{2}-1\right)^{\mu / 2}} P_{-\frac{1}{2}+t}^{\mu}(y) d y \\
=\sqrt{\frac{2}{\pi}} x^{\mu-\frac{1}{2}} \int_{\sigma-i \infty}^{\sigma+i \infty} \psi(t) K_{t}(x) d t, \quad \operatorname{Re} \mu<1 .
\end{aligned}
$$

by evaluating the $y$-integral, $[6, p .179(1)]$.

3. The main results and special cases.

Theorem 1 .

$$
\text { Let } \begin{aligned}
f(y) & =0\left(e^{-y}\right), & \text { as } y \rightarrow \infty \\
& =0(1), & \text { as } y \rightarrow 1+
\end{aligned}
$$

If

then

$$
f(y)=\frac{i}{2 \pi} \int_{\sigma-i \infty}^{\sigma+i \infty} t \sin (\pi t) \Gamma\left(\frac{1}{2}-\mu-t\right) \Gamma\left(\frac{1}{2}-\mu+t\right) P_{-\frac{1}{2}+t}^{\mu}(y) F(t) d t
$$

$$
F(t)=\int_{1}^{\infty} f(y) P_{-\frac{1}{2}+t}^{\mu}(y) d y
$$

where $t=\sigma+i \tau, \quad-\infty<\tau<\infty, \quad|\sigma|<\frac{1}{2}-\mu, \quad \operatorname{Re} \mu<1$.

Proof. Using the estimates (2.3) and (2.4) of the function $P_{-\frac{1}{2}+t}^{\mu}(y)$, along with the conditions imposed on $f$, the integral in (3.2) defining the function $g$, exists. Note that in order to ensure the existence of the integral in (3.1), representing the function $f$, one must have $t^{l-2 \mu} F(t) \in L(\sigma-i \infty, \sigma+i \infty)$ at least.

Now let,

$$
\phi(t) \equiv \frac{\pi}{2} \Gamma\left(\frac{1}{2}-\mu-t\right) \Gamma\left(\frac{1}{2}-\mu+t\right) F(t)
$$

then from (3.1) 


$$
f(y)=\frac{i}{\pi^{2}} \int_{\sigma-i \infty}^{\sigma+i \infty} t \sin (\pi t) \phi(t) P_{-\frac{1}{2}+t}^{\mu}(y) d t .
$$

Next, we define an integral operator.

$$
L f \equiv L_{\mu}[f(y) ; y \rightarrow x] \equiv \int_{1}^{\infty} \frac{e^{-x y}}{\left(y^{2}-1\right)^{\mu / 2}} f(y) d y, \quad \operatorname{Re} \mu<1 .
$$

Note that this is a linear, self adjoint operator, a sort of generalized incomplete Laplace operator. Now applying this operator to both sides of the equation (3.4) above, we obtain

due to lemma 4.

$$
\begin{aligned}
\int_{1}^{\infty} \frac{e^{-x y}}{\left(y^{2}-1\right)^{\mu / 2}} f(y) d y & =\frac{i}{\pi^{2}} \int_{1}^{\infty} \frac{e^{-x y}}{\left(y^{2}-1\right)^{\mu / 2}} d y \int_{\sigma-i \infty}^{\sigma+i \infty} t \sin (\pi t) \phi(t) \cdot P_{-\frac{1}{2}+t}^{\mu}(y) d t \\
& =\frac{i}{\pi^{2}} \sqrt{\frac{2}{\pi}} x^{\mu-\frac{1}{2}} \int_{\sigma-i \infty}^{\sigma+i \infty} t \sin (\pi t) \phi(t) K_{t}(x) d t,
\end{aligned}
$$

Or,

$$
\psi(x) \equiv \sqrt{\frac{\pi}{2}} x^{\frac{1}{2}-\mu} \int_{1}^{\infty} \frac{e^{-x y}}{\left(y^{2}-1\right)^{\mu / 2}} f(y) d y=\frac{i}{\pi^{2}} \int_{\sigma-i \infty}^{\sigma+i \infty} t \sin (\pi t) \phi(t) K_{t}(x) d t,
$$

whence, according to lemma 3 , we have the inversion,

$$
\phi(t)=\int_{0}^{\infty} \frac{\psi(v)}{v} K_{t}(v) d v,
$$

provided the integral exists. Also,

$$
\begin{aligned}
\phi(t) & =\sqrt{\frac{\pi}{2}} \int_{0}^{\infty} K_{t}(v) v^{-\frac{1}{2}-\mu} d v \int_{1}^{\infty} \frac{e^{-v y}}{\left(y^{2}-1\right)^{\mu / 2}} f(y) d y \\
& =\sqrt{\frac{\pi}{2}} \int_{1}^{\infty} \frac{f(y)}{\left(y^{2}-1\right)^{\mu / 2}} d y \int_{0}^{\infty} e^{-v y} v^{-\frac{1}{2}-\mu} K_{t}(v) d v .
\end{aligned}
$$

The change of order of integration can be justified due to absolute convergence, by making use of the estimates of the Macdonald's function $K_{t}(v)$ along with the conditions imposed on the function $f$. Now the v-integral can be evaluated, [6,p.198(27)], to give,

$$
\phi(t)=\frac{\pi}{2} \Gamma\left(\frac{1}{2}-\mu-t\right) \Gamma\left(\frac{1}{2}-\mu+t\right) \int_{1}^{\infty} f(y) P_{-\frac{1}{2}+t}^{\mu}(y) d y .
$$

And from (3.3), we have finally,

$$
F(t)=\int_{1}^{\infty} f(y) P_{-\frac{1}{2}+t}^{\mu}(y) d y
$$

as required.

Thus we have a symmetric transformation, in that, if

then

$$
f(y)=\frac{i}{2 \pi} \int_{\sigma-i \infty}^{\sigma+i \infty} t \sin (\pi t) \Gamma\left(\frac{1}{2}-\mu-t\right) \Gamma\left(\frac{1}{2}-\mu+t\right) P_{-\frac{3}{2}+t}^{\mu}(y) F(t) d y,
$$

$$
F(t)=\int_{1}^{\infty} f(y) P_{-\frac{1}{2}+t}^{\mu}(y) d y,
$$

defining a more general form of the Mehler-Fock transform of order $\mu$ and arbitrary index $-\frac{1}{2}+t$, where $t=\sigma+i \tau,-\infty<\tau<\infty$. 
Corollary: If the function $f$, satisfies the conditions of the above theorem, then

$$
f(y)=\frac{i}{2 \pi} \int_{\sigma+i \infty}^{\sigma+i \infty} \int_{1}^{\infty} t \sin (\pi t) \Gamma\left(\frac{1}{2}-\mu-t\right) \Gamma\left(\frac{1}{2}-\mu+t\right) P_{-\frac{1}{2}+t}^{\mu}(y) P_{-\frac{1}{2}+t}^{\mu}(u) f(u) d u d t .
$$

Note that from the definition of $g$, in (3.3), we have

$$
g(-t)=g(t)
$$

Now we shall look at some of the special cases.

Let $\sigma=0$. Then $(3.1)$ reduces to

Hence

$$
\begin{aligned}
f(y) & =\frac{i}{2 \pi} \int_{-i \infty}^{i \infty} t \sin (\pi t) \Gamma\left(\frac{1}{2}-\mu-t\right) \Gamma\left(\frac{1}{2}-\mu+t\right) P_{-\frac{1}{2}+t}^{\mu}(y) F(t) d t \\
& =\frac{1}{2 \pi} \int_{-\infty}^{\infty} \tau \sinh (\pi \tau) \Gamma\left(\frac{1}{2}-\mu-i \tau\right) \Gamma\left(\frac{1}{2}-\mu+i \tau\right) P_{-\frac{1}{2}+i \tau}^{\mu}(y) F(i \tau) d \tau .
\end{aligned}
$$

$$
f(y)=\frac{1}{\pi} \int_{0}^{\infty} \tau \sinh (\pi \tau) \Gamma\left(\frac{1}{2}-\mu-i \tau\right) \Gamma\left(\frac{1}{2}-\mu+i \tau\right) P_{-\frac{1}{2}+i \tau}^{\mu}(y) F(i \tau) d \tau,
$$

using the properties (2.1) and (3.6),

where $F(i \tau)=\int_{1}^{\infty} f(y) P_{-\frac{3}{2}+i \tau}^{\mu}(y) d y$,

giving us a generalized Mehler-Fock transformation [3] .

Further if we put $\mu=0$, then the above pair is reduced to

and

$$
f(y)=\int_{0}^{\infty} \tau \operatorname{coth}(\pi \tau) P_{-\frac{1}{2}+i \tau}(y) F(i \tau) d \tau
$$

$$
F(i \tau)=\int_{1}^{\infty} f(y) P_{-\frac{1}{2}+i \tau}(y) d y
$$

the usual Mehler-Fock transformation of zero order [2, p.389]. Next, we shall derive, formally, a Parseval type relation. Let us define the function $F(t)$ and $G(t)$ to be the generalized Mehler-Fock transforms of the function $f(y)$ and $g(y)$ respectively, so that

and

$$
F(t)=\int_{1}^{\infty} f(y) P_{-\frac{1}{2}+t}^{\mu}(y) d y
$$

$$
G(t)=\int_{1}^{\infty} g(y) P_{-\frac{1}{2}+t}^{\mu}(y) d y
$$

where $t=\sigma+i \tau,|\sigma|<\frac{1}{2}$, $\operatorname{Re} \mu<1$ and $-\infty<\tau<\infty$. And of course, as proved above

$$
f(y)=\frac{i}{2 \pi} \int_{\sigma-i \infty}^{\sigma+i \infty} t \sin (\pi t) \Gamma\left(\frac{1}{2}-\mu-t\right) \Gamma\left(\frac{1}{2}-\mu+t\right) P_{-\frac{1}{2}+t}^{\mu}(y) F(t) d t,
$$

and a similar formula for $g(y)$.

Consider the integral,

$$
\begin{gathered}
\frac{i}{2 \pi} \int_{\sigma-i \infty}^{\sigma+i \infty} t \sin (\pi t) \Gamma\left(\frac{1}{2}-\mu-t\right) \Gamma\left(\frac{1}{2}-\mu+t\right) F(t) G(t) d t \\
\equiv \frac{i}{2 \pi} \int_{\sigma-i \infty}^{\sigma+i \infty} \psi(t) F(t) G(t) d t \text {, say }
\end{gathered}
$$




$$
\begin{aligned}
& =\frac{i}{2 \pi} \int_{\sigma-i \infty}^{\sigma+i \infty} \psi(t) F(t) \dot{x} t \int_{1}^{\infty} g(y) P_{-\frac{1}{2}+t}^{\mu}(y) d y \\
& =\frac{i}{2 \pi} \int_{1}^{\infty} g(y) d y \int_{\sigma-i \infty}^{\sigma+i \infty} \psi(t) F(t) P_{-\frac{1}{2}+t}^{\mu}(y) d y \\
& =\int_{1}^{\infty} g(y) f(y) d y .
\end{aligned}
$$

Thus we have,

Theorem 2 .

Let $F$ and $G$ be the generalized Mehler-Fock transforms of $f$ and $g$. Then,

$$
\begin{gathered}
\frac{i}{2 \pi} \int_{\sigma-i \infty}^{\sigma+i \infty} t \sin (\pi t) \Gamma\left(\frac{1}{2}-\mu-t\right) \Gamma\left(\frac{1}{2}-\mu+t\right) F(t) G(t) d t \\
\quad=\int_{1}^{\infty} f(y) g(y) d y
\end{gathered}
$$

This is a formal derivation, but the analysis can be justified by absolute convergence, using suitable conditions on the functions involved. If we set $\sigma=0$ in (3.7), we can easily deduce that

$$
\begin{gathered}
\frac{1}{\pi} \int_{0}^{\infty} \tau \sinh (\pi \tau) \Gamma\left(\frac{1}{2}-\mu-i \tau\right) \Gamma\left(\frac{1}{2}-\mu+i \tau\right) F(i \tau) G(i \tau) d \tau \\
=\int_{1}^{\infty} f(y) g(y) d y,
\end{gathered}
$$

a known result [3]. If, further, we put $\mu=0$, then (3.8) reduces to [2, p.394],

where,

$$
\frac{1}{\pi i} \int_{0}^{\infty} \tau \tanh (\pi \tau) F(i \tau) G(i \tau) d \tau=\int_{1}^{\infty} f(y) g(y) d y
$$

$$
F(i \tau)=\int_{1}^{\infty} f(y) P_{-\frac{1}{2}+i}^{\mu}(y) d y
$$

and a similar representation for $G\left(i_{\tau}\right)$.

Although the functions $F$ and $f$ satisfying (3.1) and (3.2) are defined for restricted values of $\mu$, one can formally extend the results as follows:

If

then

$$
f(y)=\frac{i}{2 \pi} \int_{\sigma-i \infty}^{\sigma+i \infty} t \sin (\pi t) \Gamma\left(\frac{1}{2}-m-t\right) \Gamma\left(\frac{1}{2}-m+t\right) P_{-\frac{1}{2}+t}^{m}(y) F(t) d t,
$$

$$
F(t)=\int_{1}^{\infty} f(y) P_{-\frac{1}{2}+t}^{m}(y) d y
$$

where $t=\sigma+i \tau,|\sigma|<\frac{1}{2},-\infty<\tau<\infty$ and $m=0,1,2, \ldots$. And all the results derived above hold. For instance, if $\sigma=0$, then we have from above,

where

$$
f(y)=(-1)^{m} \int_{0}^{\infty} \tau \tanh (\pi \tau) \frac{\Gamma\left(\frac{1}{2}-m+i \tau\right)}{\Gamma\left(\frac{1}{2}+m+i \tau\right)} P_{-\frac{1}{2}+i \tau}^{m}(y) F(i \tau) d \tau
$$

$$
F(i \tau)=\int_{1}^{\infty} f(y) P_{-\frac{1}{2}+i \tau}^{m}(y) d y \text {. }
$$


If we let
\[ \widetilde{\widetilde{J}}_{m}(\tau) \equiv \frac{\Gamma\left(\frac{1}{2}-m+i \tau\right)}{\Gamma\left(\frac{1}{2}+m+i \tau\right)} F(i \tau), \]

then

$$
\widetilde{f}_{m}(\tau)=\int_{1}^{\infty} f(y) \frac{\Gamma\left(\frac{1}{2}-m+i \tau\right)}{\Gamma\left(\frac{1}{2}+m+i \tau\right)} P_{-\frac{1}{2}+i \tau}^{m}(y) d y,
$$

$$
\widetilde{f}_{m}(\tau)=\int_{0}^{\infty} f(y) P_{-\frac{i}{2}+i \tau}^{-m}(y) d y
$$

using the property (2.2), where

$$
f(y)=(-1)^{m} \int_{0}^{\infty} \tau \tanh (\pi \tau) P_{-\frac{1}{2}+i \tau}^{m}(y) \widetilde{f}_{m}(\tau) d \tau
$$

giving us a pair of Mehler-Fock transform of order $m$, [2, p.416]. It may be pointed out here that one can prove Lemma 4, using the result of Theorem 1 . In other words, assume (3.1) and (3.2) hold one can show that if

then

$$
f(v)=\frac{i}{\pi^{2}} \cdot \int_{\sigma-i \infty}^{\sigma+i \infty} z \sin (\pi z) K_{z}(v) F(z) d z
$$

$$
F(z)=\int_{0}^{\infty} \frac{f(v)}{v} K_{z}(v) d v, \quad|\sigma|<\frac{1}{2}
$$

the so called generalized Kontrovich-Lebedev transform. Cosequently one can say that there is an equivalence between the generalized Mehler-Fock and generalized KontrovichLebedev transform.

A slightly different form of the Mehler-Fock transformation and its inversion, can easily be established, by making use of the pair of Kontrovich-Lebedev transforms $[3$, p. 75(76)],

and

$$
f(x)=\frac{1}{\pi_{i}} \int_{\sigma-i_{\infty}}^{\sigma+i \infty} t K_{t}(x) g(t) d t
$$

That is,

$$
g(t)=\int_{0}^{\infty} \frac{f(v)}{v} I_{t}(v) d v
$$

Theorem 3 .

$$
\text { If }
$$

then

$$
f(y)=\frac{1}{\pi i} \int_{\sigma-i \infty}^{\sigma+i \infty} z g(z) P_{-\frac{3}{2}+z}^{\mu}(y) d z, \quad y \in[1, \infty)
$$

$$
g(z)=e^{i \mu \pi} \int_{1}^{\infty} f(v) Q_{-\frac{z_{2}}{2}+z}^{-\mu}(v) d v, \quad \operatorname{Re} \mu<1, \quad|\sigma|<\frac{1}{2}
$$

where $P_{\nu}^{\mu}$ and $Q_{\nu}^{\mu}$ are the associated Legendre functions of first and second kind respective1y.

Alternatively,

$$
f(y)=\frac{e^{i \mu \pi}}{\pi i} \int_{\sigma-i \infty}^{\sigma+i \infty} \int_{1}^{\infty} z P_{-\frac{1}{2}+z}^{\mu}(y) Q_{-\frac{1}{2}+z}^{-\mu}(v) d v d z
$$

The proof of the above theorem is on the same lines as the proof of Theorem 1 , the linear operator used in the proof will now be 


$$
L[f(y) ; y \rightarrow x] \equiv \int_{0}^{\infty} e^{-x y} y^{\mu-\frac{1}{2}} f(y) d y,
$$

which on applying to the equation (3.13), will reduce it to the form (3.11). Then using the inversion (3.10), will produce equation ( 3.14 ), as desired.

\section{REFERENCES}

1. ERDELYI, A. et al. Higher Transcendental Functions Vol. I, Bateman Manuscript Project, McGraw-Hill Book Co., New York, 1953.

2. SNEDDON, I.N. The Use of Integral Transforms, McGraw-Hill Book Co., New York, 1972.

3. LOWNDES, J.S. Note on Generalized Mehler-Fock Transform, Proc. Camb. Philos. Soc. 60 (1964), 57-59.

4. ZEMANIAN, A.H. The Kontorovich-Lebedev Transformation on Distributions of Compact Support and Its Inversion, Math. Proc. Cambridge Philos. Soc. 77 (1975), 139-143.

5. ERDELYI, A. et al. Higher Transcendental Functions Vol. II, Bateman Manuscript Project, McGraw-Hill Co., New York, 1953.

6. ERDELYI, A. Tables of Integral Transforms Vol. I, Bateman Manuscript Project, McGraw-Hill Book Co., New York, 1953. 


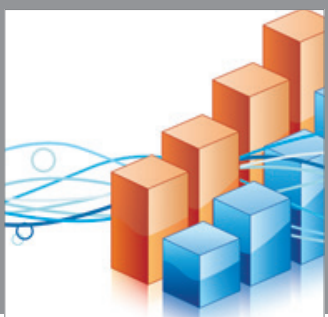

Advances in

Operations Research

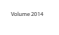

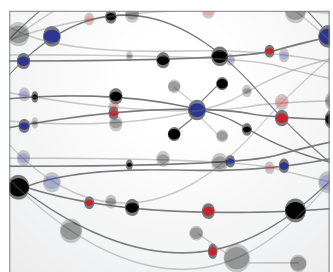

\section{The Scientific} World Journal
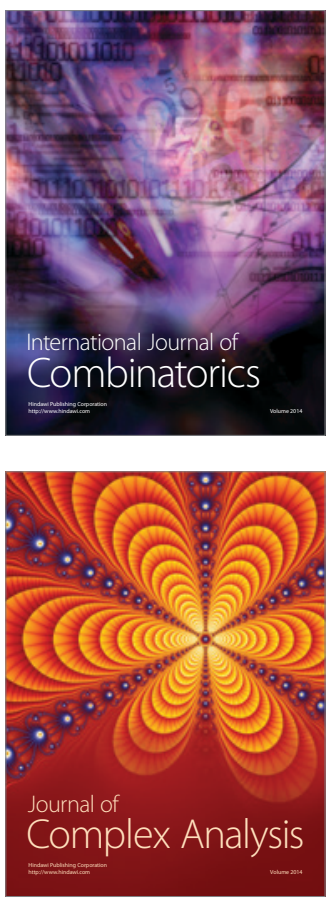

International Journal of

Mathematics and

Mathematical

Sciences
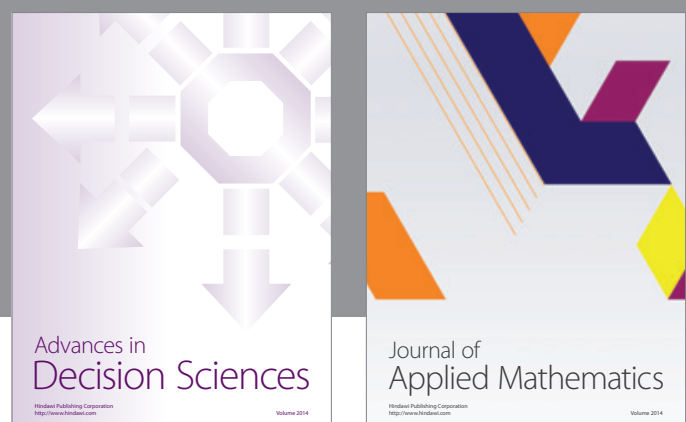

Journal of

Applied Mathematics
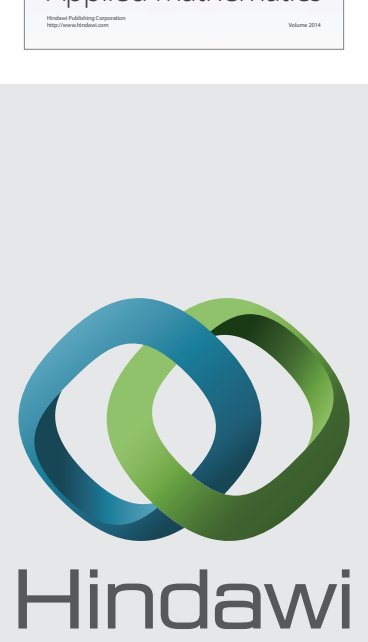

Submit your manuscripts at http://www.hindawi.com
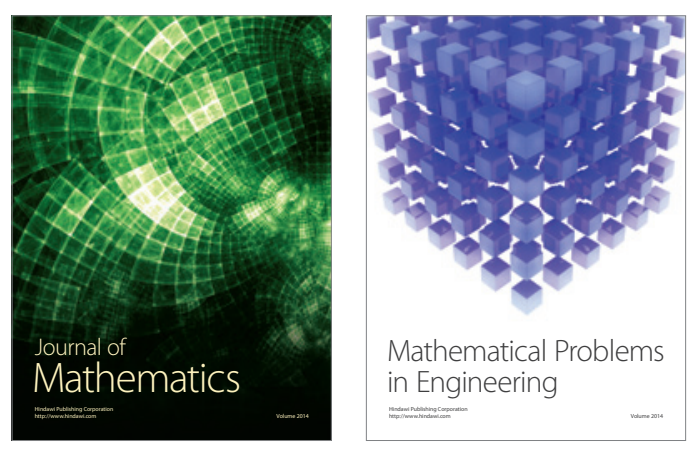

Mathematical Problems in Engineering
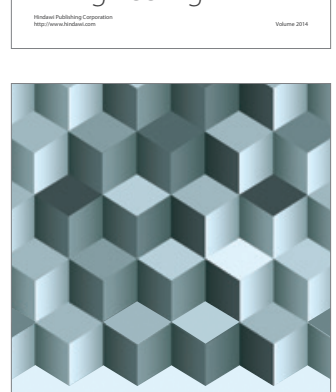

Journal of

Function Spaces
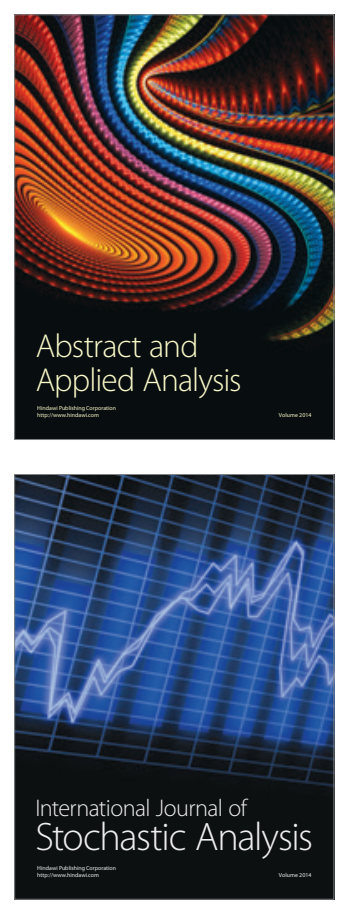

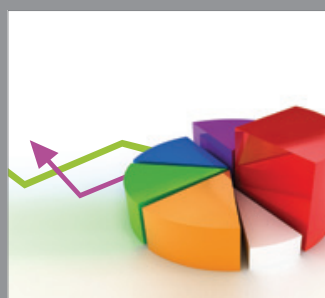

ournal of

Probability and Statistics

Promensencen
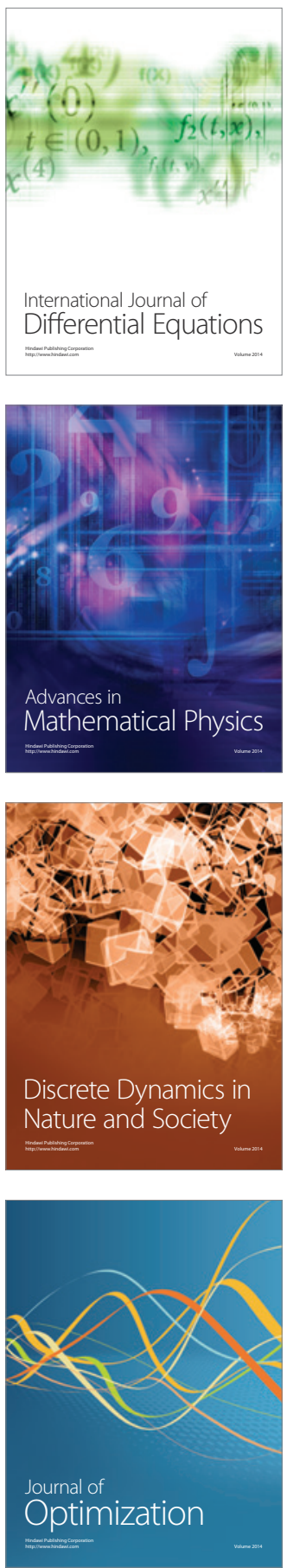